\title{
Blind Near-MAP Selection Diversity with Multi-User Detection for Multi-User OFDM
}

\author{
Yu-Yi Cheng Yumin Lee Hsueh-Jyh Li \\ Graduate Institute of Communication Engineering \\ National Taiwan University \\ Taipei 10617, Taiwan
}

\begin{abstract}
In this paper, a receiver algorithm for OFDM with receiver diversity is proposed. The proposed receiver uses a new multi-user generalization of the maximum a posteriori probability (MAP) selection diversity combining followed by successive interference cancellation with least-square projection for multi-user detection. Simulation results show that the proposed approach achieves near-maximal-ratiocombining performance with much lower complexity.
\end{abstract}

\section{INTRODUCTION}

Orthogonal frequency division multiplexing (OFDM) [1] is an efficient transmission technique for high-rate wireless data communication systems. Although OFDM is very effective in combating the effect of delay-spread, it stills suffers from signal fading caused by multipath propagation as well as interference caused by other users in a multi-user scenario. Receiver diversity [2] is a technique for mitigating both effects. By properly combining the signals from multiple receiving antennas, a diversity receiver can greatly enhance the performance of an OFDM system in the presence of fading and interference. Conventional receiver diversity techniques [3] include maximal ratio combining (MRC), equal-gain combining (EGC), and selection diversity combining (SDC). MRC linearly combines the received signal using weights proportional to the signal-to-noise ratio (SNR). In general, MRC has the best performance and the highest computational complexity. On the other hand, SDC selects the "best" diversity branch according to some selection metric, and has lower complexity and slightly inferior performance compared to MRC. The selection metric is crucial for SDC. In [4], selection metrics based on the pre-processed signal amplitude are proposed for single-user transmission systems. The proposed metrics

This research work was supported by the National Science Council of the Republic of China under grant number NSC 91-2219-E-002-023. are very simple, but cannot be applied in multi-user systems directly. In [5], a selection metric that is optimal in the maximum $a$ posteriori probability (MAP) sense was proposed for simple single-carrier, single-user transmission over multipath fading channels. The MAP selection metrics are simple to compute and achieves a performance that is close to MRC. Finally, the performance of EGC is in general inferior to both MRC and SDC. We therefore focus only on MRC and $\mathrm{SDC}$ in this paper.

In this paper, we generalize the previously proposed [5] MAP SDC scheme to a multi-user OFDM diversity receiver. There are several features in the proposed receiver. First, approximate MAP SDC is performed on a per-subcarrier basis using novel selection metrics that will be derived later. Second, computation of the selection metrics requires accurate estimates of the channel between all users and receiver antennas. In this paper, we use the two-step subspace-based multi-user blind channel estimation algorithm proposed in [6] to derive the channel estimates. Third, after per-subcarrier SDC is completed, the transmitted signals from each user are separated using a new recursive algorithm referred to as successive interference cancellation with least-squares projection (SIC-LSP), which will also be described later in detail. Since no pilots are used, the receiver is completely blind. Simulation results show that the proposed algorithm achieves very good performance. In particular, with the same blind channel estimates, the proposed algorithm performs closely to per-subcarrier MRC, which is much more complex.

\section{SySTEM BLOCK DiAgRAM}

Consider the multi-user OFDM transmission system shown in Fig. 1, in which $S$ quasi-synchronous users transmit independent and identically distributed OFDM symbols in the same bandwidth at the same time. For the $s$-th user, information bits are convolutionally encoded and mapped onto quaternary phase-shift keying (QPSK) 
subsymbols, which are then serial-to-parallel converted and processed by the $N$-point inverse discrete Fourier transformer (IDFT), where $N$ is the number of subcarriers. The output of IDFT is parallel-to-serial converted and a cyclic prefix (CP) of length $N_{C P}$ is inserted to obtain a discrete-time signal $x_{s, l}$. For simplicity, we assume that $\mathrm{CP}$ is sufficiently long so that there is no IBI. The continuous-time transmitted signal of user $s$ is given by

$$
x_{s}(t) \equiv \sum_{l} x_{s, l} q(t-l T),
$$

where $T$ is the sample time of the OFDM symbol, and $q(\bullet)$ is the pulse-shaping function. The user signals are transmitted to $M$ receiving antennas through independent wireless channels, each modeled as a modified Jakes' frequency-selective multipath fading channel corrupted by additive white Gaussian noise (AWGN) [7].

At the receiver, the signals $u^{(m)}(t)$ received from the $m$-th antennas $(m=0, \ldots, M-$ 1) are each filtered by a lowpass filter and sampled at a rate of $P / T$ samples per second, resulting in a discrete-time signal given by

$u_{n, p}^{(m)} \equiv u^{(m)}\left(n T+\frac{p T}{P}\right)=\sum_{s=0}^{S-1} \sum_{b=0}^{L-1} x_{s, n-b} h_{b, s, p}^{(m)}+v_{n, p}^{(m)}$

for $m=0,1, \ldots, M-1, p=0,1, \ldots, P-1$, and $n=0,1$, $2, \ldots$, where $h$ is the poly-phase discrete-time equivalent channel and $L$ is the channel memory. The signal $u_{n, p}^{(m)}$ is then fed into the proposed receiver to be discussed in the next section.

\section{PROPOSED RECEIVER AlgORITHM}

A block diagram for the proposed receiver is shown in Fig. 1. The proposed receiver consists of the two-step blind channel estimator proposed in [6], SDC or MRC diversity combiner, and SIC-LSP. The detailed block diagrams for SDC and SIC-SLP are shown in Figs. 2 and 3. The channel estimator provides channel estimates for computing the selection metrics for the SDC or the combining weightings for $\mathrm{MRC}$, both of which operate in the frequency domain on a per-phase per-subcarrier basis. SDC uses a new approximate MAP selection metric to be described later. The selected output is processed by the SIC-LSP algorithm for user separation. Finally, the output of SIC-LSP for each user is convolutionally decoded to obtain the user information bits.

\section{A. Apporximate MAP SDC Metric}

Mathematically, the time-domain input to the receiver can be expressed as an $N P$-dimensional poly-phase vector given by

$$
\mathbf{u}^{(m)} \equiv\left[\begin{array}{lll}
\mathbf{u}_{0}^{(m) T} & \cdots & \mathbf{u}_{P-1}^{(m) T}
\end{array}\right]^{T},
$$

where

$$
\mathbf{u}_{p}^{(m)}=\left[\begin{array}{lll}
u_{n, p}^{(m)} & \cdots & u_{n-N+1, p}^{(m)}
\end{array}\right]^{T}
$$

The $N$ components in $\mathbf{u}_{p}^{(m)}$ are $T$-spaced samples of a particular OFDM symbol excluding $\mathrm{CP}$ with sampling offset $p T / P$. By first taking the DFT of $\mathbf{u}_{p}^{(m)}$ to obtain $\mathbf{U}_{p}^{(m)}$ and letting

$$
\mathbf{U}^{(m)}=\left[\begin{array}{llll}
\mathbf{U}_{0}^{(m) T} & \mathbf{U}_{1}^{(m) T} & \ldots & \mathbf{U}_{P-1}^{(m) T}
\end{array}\right]^{T},
$$

we have

$$
\mathbf{U}^{(m)}=\mathbf{H}^{(m)} \mathbf{X}+\mathbf{V}^{(m)},
$$

where

$$
\mathbf{X}=\left[\begin{array}{lll}
\mathbf{X}_{0}^{T} & \cdots & \mathbf{X}_{S-1}^{T}
\end{array}\right]^{T}
$$

in which

$$
\mathbf{X}_{s}=\left[\begin{array}{lll}
X_{0, s} & \cdots & X_{N-1, s}
\end{array}\right]^{T}
$$

is the vector of transmitted subsymbols of the $s$-th user, and $\mathbf{V}^{(m)}$ is a circularly symmetric complex Gaussian random vector. The matrix $\mathbf{H}^{(m)}$ is the multi-user poly-phase frequency-domain channel matrix of the $m$-th antenna that has $P$ block rows and $S$ block columns. The $(p, s)$-th block element of $\mathbf{H}^{(m)}$ is an $N \times N$ diagonal matrix whose diagonal entries are the DFT values of $\mathbf{h}_{s, p}^{(m)}=\left[\begin{array}{lll}h_{0, s, p}^{(m)} & \cdots & h_{L-1, s, p}^{(m)}\end{array}\right]^{T}$. Let $H_{k, s, p}^{(m)}, k$ $=0, \ldots, N-1$, be estimates of these diagonal entries (frequency-domain channel estimates) obtained using the previously proposed two-step blind channel estimator, we have

$$
U_{k, p}^{(m)} \approx \sum_{s=0}^{S-1} H_{k, s, p}^{(m)} X_{k, s}+V_{k, p}^{(m)},
$$

where $V_{k, p}{ }^{(m)}$ is the frequency-domain Gaussian noise.

The operation of the proposed per-phase per-subcarrier SDC is as follows. For a particular subcarrier $k$, SDC selects one antenna for each sampling phase $p$. The antennas are chosen so that the a posteriori probability of correct joint detection for the $k$-th sub-symbol of all $S$ users conditioned solely on the output of the chosen antennas for sampling phases $0 \ldots P-1$ is maximized. Mathematically, Let $m_{k, p}$ denote the antenna element selected for the $k$-th subcarrier at sampling phase $p$, we let

$$
\left(\begin{array}{lll}
m_{k, 0} & \cdots & m_{k, P-1}
\end{array}\right) \equiv \underset{l_{0} \ldots l_{P-1}}{\arg \max } P_{k}\left(l_{0} \cdots l_{P-1}\right)
$$

where 


$$
\begin{aligned}
& P_{k}\left(l_{0} \cdots l_{P-1}\right) \equiv \\
& \operatorname{Pr}\left[\text { Corr. Det. of } X_{k, 0} \cdots X_{k, S-1} \mid \begin{array}{l}
U_{k, 0}^{\left(l_{0}\right)} \cdots U_{k, P-1}^{\left(l_{P-1}\right)} \\
\text { were observed }
\end{array}\right] .
\end{aligned}
$$

Using Bayes' rule and assuming equally likely transmitted sub-symbols, we have

$$
\begin{aligned}
& P_{k}\left(l_{0} \cdots l_{P-1}\right)= \\
& \frac{K_{1} \exp \left(-\frac{1}{2}(\mathbf{a}-\mathbf{B} \hat{\mathbf{x}})^{H} \mathbf{R}_{V}^{-1}(\mathbf{a}-\mathbf{B} \hat{\mathbf{x}})\right)}{K_{2} \sum_{\text {all possible } \mathbf{x}} \exp \left(-\frac{1}{2}(\mathbf{a}-\mathbf{B} \mathbf{x})^{H} \mathbf{R}_{V}^{-1}(\mathbf{a}-\mathbf{B} \mathbf{x})\right)},
\end{aligned}
$$

where

$$
\mathbf{a} \equiv\left[U_{k, 0}^{\left(l_{0}\right)} \cdots U_{k, P-1}^{\left(l_{P-1}\right)}\right]^{T}
$$

is the vector of observations, $\mathbf{B}$ is a $P \times S$ matrix whose $(p, s)$-entry is $H_{k, s, p}^{\left(l_{p}\right)}$,

$$
\mathbf{x}=\left[X_{k, 0} \ldots X_{k, S-1}\right]
$$

is the vector of transmitted $k$-th sub-symbol of all users, $\mathbf{R}_{V}$ is the covariance matrix of the noise component of $\mathbf{a}, \hat{\mathbf{x}}$ is the detection result based on the observation a, and $K_{1}$ and $K_{2}$ are appropriate scaling constants. For the sake of brevity, the dependencies of the quantities in the right hand side of (12) on $k$, and $l_{0}, \ldots, l_{P-1}$ are not explicitly shown.

In principle, for a given $k, P_{k}\left(l_{0}, \ldots, l_{P-1}\right)$ can be evaluated using (12) for all possible combinations of $l_{0} \ldots l_{P-1}$, and the combination corresponding to the maximum result is chosen by SDC. In practice, however, this procedure is computationally impractical. In order to simplify (12), we make the following approximations. First, we assume that $\mathbf{R}_{V}=$ $\sigma_{V}{ }^{2} \mathbf{I}_{P}$, where $\mathbf{I}_{P}$ is the $P \times P$ identity matrix. Second, we approximate the mixture of Gaussian functions in the denominator of (12) as a single Gaussian function given by

$$
\left(2 \pi \prod_{p=0}^{P-1} \sigma_{p}^{2}\left(l_{p}\right)\right)^{-\frac{P}{2}} \exp \left(-\frac{\mathbf{a}^{H} \mathbf{R}^{-1} \mathbf{a}}{2}\right),
$$

where $\mathbf{R}$ is a diagonal matrix whose $p$-th diagonal entry is given by

$$
\sigma_{p}^{2}\left(l_{p}\right) \equiv \sigma_{V}^{2}+\sum_{s=0}^{S-1} \sigma_{s}^{2}\left|H_{k, s, p}^{\left(l_{p}\right)}\right|^{2}
$$

in which $\sigma_{\mathrm{s}}{ }^{2}$ is the average signal sub-symbol energy of the $s$-th user. And finally, we approximate the Gaussian function in the numerator of (12) as $1 / \sigma_{V}$. These approximations can be justified as follows. The first approximation states that the noise component in the observation $\mathbf{a}$ is white, which is typically the case for thermal noise. The second approximation holds if the number of possible values of $\mathbf{x}$ is sufficiently large. This is typically true even in a system with only a handful of users with moderately sized signal constellations, because the number of possible values of $\mathbf{x}$ is exponentially related to the cardinality of the signal constellations. Finally, the third approximation holds if the noise is statistically small and the detection result is sufficiently accurate, so that $\mathbf{a}-\mathbf{B} \hat{\mathbf{x}}$ is close to zero with high probability. The approximate MAP SDC rule is therefore given by

$$
\left(\begin{array}{lll}
m_{k, 0} & \cdots & m_{k, P-1}
\end{array}\right) \equiv \underset{l_{0} \cdots l_{P-1}}{\arg \min } \Delta_{k}\left(l_{0} \cdots l_{P-1}\right)
$$

where

$$
\Delta_{k}\left(l_{0}, \cdots l_{P-1}\right) \equiv \sum_{p=0}^{P-1} \frac{\left|U_{k, p}^{\left(l_{p}\right)}\right|^{2}}{\sigma_{V}^{2}+\sum_{s=0}^{S-1} \sigma_{s}^{2}\left|H_{k, s, p}^{\left(l_{p}\right)}\right|^{2}}
$$

It will be shown by simulation that (17) and (18) indeed provide very good results.

B. Successive Interference Cancellation With Least-Squares Projection (SIC-LSP)

As shown in Fig. 2, the output of SDC is processed by SIC-LSP for multi-user detection. Mathematically, the output of SDC is a $P$-dimension vector given by

$$
\mathbf{U}_{k}=\left[\begin{array}{llll}
U_{k, 0}^{\left(m_{k, 0}\right)} & U_{k, 1}^{\left(m_{k, 1}\right)} & \cdots & U_{k, P-1}^{\left(m_{k, P-1}\right)}
\end{array}\right]^{T}
$$

where $m_{k, 0} \ldots m_{k, P-1}$ are obtained using (17). The vector $\mathbf{U}_{k}$ is related to the transmitted $k$-th sub-symbols by

$$
\mathbf{U}_{k}=\sum_{s=0}^{S-1} \mathbf{H}_{k, s} X_{k, s}+\mathbf{V}_{k},
$$

where

$$
\mathbf{H}_{k, s}=\left[\begin{array}{llll}
H_{k, s, 0}^{\left(m_{k, 0}\right)} & H_{k, s, 1}^{\left(m_{k, 1}\right)} & \cdots & H_{k, s, P-1}^{\left(m_{k, P-1}\right)}
\end{array}\right]^{T}
$$

and

$$
\mathbf{V}_{k}=\left[\begin{array}{llll}
V_{k, 0}^{\left(m_{k, 0}\right)} & V_{k, 1}^{\left(m_{k, 1}\right)} & \cdots & V_{k, P-1}^{\left(m_{k, P-1}\right)}
\end{array}\right]^{T} .
$$

The task of SIC-LSP is to detect $X_{k, 0} \ldots X_{k, S-1}$ based on $\mathbf{U}_{k}$. In the proposed receiver, this is done by first computing the least-squares (orthogonal) projection of $\mathbf{U}_{k}$ onto the span of $\left\{\mathbf{H}_{k, 1} \ldots \mathbf{H}_{k, S-1}\right\}$ given by

$$
\mathbf{E}_{k, 1} \equiv \mathbf{A}_{k, 1}\left(\mathbf{A}_{k, 1}^{H} \mathbf{A}_{k, 1}\right)^{-1} \mathbf{A}_{k, 1}^{H} \mathbf{U}_{k}
$$

where $\mathbf{A}_{k, \mathrm{~s}}$ is a $P \times(S-s)$ matrix whose columns are $\mathbf{H}_{k, s} \ldots \mathbf{H}_{k, S-1}$. We next subtract off the projection $\mathbf{E}_{k, 1}$ from $\mathbf{U}_{k}$ to compute

$$
Z_{k, 0} \equiv \frac{1}{\left\|\mathbf{H}_{k, 0}\right\|^{2}} \mathbf{H}_{k, 0}^{H}\left(\mathbf{U}_{k}-\mathbf{E}_{k, 1}\right) \cdot
$$

The detected symbol $\hat{X}_{k, 0}$ for user 0 is then obtained by appropriate slicing $Z_{k, 0}$. The remaining users are then detected by repeating the following procedure for $s=1 \ldots S-1$ :

Step 1: Compute

$$
\mathbf{U}_{k}^{\prime}=\mathbf{U}_{k}-\sum_{s^{\prime}=0}^{s-1} \mathbf{H}_{k, s^{\prime}} \hat{X}_{k, s^{\prime}}
$$


Step 2: Compute

$$
\mathbf{E}_{k, s+1} \equiv \mathbf{A}_{k, s+1}\left(\mathbf{A}_{k, s+1}^{H} \mathbf{A}_{k, s+1}\right)^{-1} \mathbf{A}_{k, s+1}^{H} \mathbf{U}_{k}^{\prime}
$$

Step 3: Compute

$$
Z_{k, s} \equiv \frac{1}{\left\|\mathbf{H}_{k, s}\right\|^{2}} \mathbf{H}_{k, s}^{H}\left(\mathbf{U}_{k}^{\prime}-\mathbf{E}_{k, s+1}\right)
$$

Step 4: Appropriately slice $Z_{k, s}$ to obtain $\hat{X}_{k, s}$.

Note that (25) is the equation for conventional SIC where the effect of the detected users (0 to $s-1)$ are synthesized and cancelled using decision feedback to obtain a cleaner signal $\mathbf{U}^{\prime}{ }_{k}$. In (26) and (27), the signal $\mathbf{U}_{k}^{\prime}$ is further cleaned up by projecting onto the span of $\mathbf{H}_{k, s}$. In Step $4, \hat{X}_{k, s}$ is obtained by slicing the projection given in (27). A block diagram for the entire procedure is given in Figure 3. Note that since no pilots are required, the proposed receiver is completely blind.

\section{SIMULATION RESULTS}

The performance of the proposed receiver algorithm is evaluated using computer simulation. User information bits are first encoded using a convolutional encoder and mapped onto $N=64$ QPSK subsymbols. $N_{C P}$ is set to 16 and a $35 \%$ roll-off square-root raised cosine function is used for pulse shaping. The receiver filters used in the simulations are also $35 \%$ roll-off square-root raised cosine filters. The carrier frequency is $900 \mathrm{MHz}$. No frequency offset is assumed between the transmitter and the receiver. Unless otherwise stated, the receiver consists of $M=2$ antennas, the signals out of which are combined using the proposed approximate MAP SDC scheme followed by an SIC-LSP multi-user detector (SDC/SIC-LSP). The wireless channels are simulated using power-delay profiles following the Typical Urban (TU) channel models [8]. It is assumed that channels are statistically independent across users and across antennas. In addition to the proposed receiver, per-subcarrier MRC followed by SIC-LSP (MRC/SIC-LSP) as well as a single-antenna receiver (no diversity) are also simulated as baselines for comparison.

The decoded bit error rate (BER) of MAP/SIC-LSP, MRC/SIC-LSP, and single-antenna (no diversity) receivers are shown in Figure 4 as functions of $E_{b} / N_{0}$ for the single-user case $(S=1)$, where the MRC combiner uses the estimated channel gains as weightings and produces an output given by

$$
U_{k, p}^{M R C}=\sum_{m=0}^{M-1} \sum_{p=0}^{P-1} H_{k, 0, p}^{(m) H} U_{k, p}^{(m)}
$$

The over-sampling factor is $P=1$ for this simulation case. Here $E_{b}$ is the energy per transmitted bit and $N_{0} / 2$ is the two-sided power spectral density of the AWGN. Note that for $S=$ 1, SIC-LSP degenerates to the conventional single-user detector. It can be seen that diversity combining results in a 2 to $3 \mathrm{~dB}$ performance gain over the single-antenna case. Furthermore, the performance of SDC is only slightly inferior to MRC. Note that for $S=P=1$ and $M=2$, SDC is only slightly less complex than MRC. Therefore although Fig. 2 justifies the concept of SDC, it does not justify replacing MRC by SDC.

The average decoded bit error rate (BER) of SDC/SIC-LSP and MRC/SIC-LSP are shown in Fig. 5(a) as functions of $E_{b} / N_{0}$ and Fig. 5 (b) as functions of the signal-to-interference ratio (SIR), where the interference signal is an OFDM signal centered at the same carrier frequency and modulated using random data. In Fig. 5(a), the SIR is fixed at $4 \mathrm{~dB}$ and in Fig. 5 (b) $E_{b} / N_{0}$ is fixed at $30 \mathrm{~dB}$. The number of active users is $S=2$. It can be seen that MRC /SIC-LSP outperforms SDC/SIC-LSP only by roughly $1 \mathrm{~dB}$. However, for $S>1$, evaluating (17) and (18) for SDC is considerably simpler than computing the combining weightings for MRC. In particular, computing the MRC weightings for each subcarrier at least requires the inversion of $S M P \times M P$ complex matrices, therefore the complexity associated with computing the weights is at least proportional to $S M^{3} P^{3}$. On the other hand, (18) can be evaluated by first computing $\left|U_{k, p}^{\left(l_{p}\right)}\right|^{2}$ and $\left|H_{k, s, p}^{\left(l_{p}\right)}\right|^{2}$ for all $s, l_{p}$, and $p$. The $M^{2}$ possible values of $\Delta_{k}(\bullet)$ are then computed from these values. The complexity associated with (18) is therefore proportional to $M^{2}+M P+S M P$. It can thus be argued that the approximate MAP SDC receiver proposed in this paper is a very attractive alternative to MRC because it sacrifices very little performance for a significant complexity reduction.

Finally, the decoded BER of SDC/SIC-LSP are shown in Figure 6 as functions of $E_{b} / N_{0}$ for $S=3$ and $P=6$. The users are assumed to have the same constellation symbol energy. Instead of showing the user-averaged decoded BER, here we show the decoded BER for each user separately. As a baseline for comparison, the performance of a receiver with SDC followed only by LSP is also shown in these figures, where the user sub-symbols are obtained by slicing their least-squares estimates obtained from the output of SDC. It can be seen that incorporating SIC into SDC brings a gain of roughly $1 \mathrm{~dB}$. 


\section{CONCLUSION}

An algorithm for OFDM with receiver diversity is proposed in this paper. The proposed receiver consists of a two-step blind channel estimator, approximate per-subcarrier per-phase MAP SDC, and SIC-LSP for multi-user detection. Simulation results show that the proposed algorithm achieves near-MRC performance at much lower complexity.

\section{REFERENCES}

[1] R.V. Nee and R. Prasad, OFDM for Wireless Multimedia Communications, Artech House, 2000.

[2] J. C. Liberti and T. S. Rappaport, Smart Antennas for Wireless Communications: IS-95 and Third Generation CDMA Application, Prentice Hall, 1999.

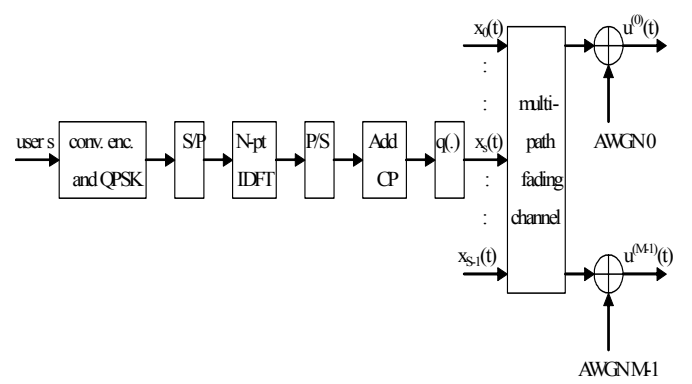

Transmitter and channel
[3] T. S. Rappaport, Wireless Communications: Principles and Practice, Prentice Hall, 1996

[4]E. A. Neasmith and N. C. Beaulieu, "New results on selection diversity," IEEE Trans. on Communications, vol.46, no.5, May 1998, pp.695 -704

[5]Y. Lee and D. C. Cox, "MAP selection -diversity DFE for indoor wireless data communications," IEEE Journal on Selected Areas in Communications, vol.16 no.8, Oct. 1998, pp. $1376-1384$

[6]Y.-Y. Cheng, Y. Lee and H.-J. Li, "Subspace-MMSE Blind Channel Estimation for Multiuser OFDM with Receiver Diversity" accepted for GLOBECOM 2003

[7]P. Dent, G.E. Bottomley, and T. Croft, "Jakes fading model revisited," Electronics Letters, vol.29, no.13, pp.1162-1163, June 1993.

[8] 3G TR 25.943: 3rd Generation Partnership Project; Technical Specification Group(TSG) RAN WG4; Deployment aspects.

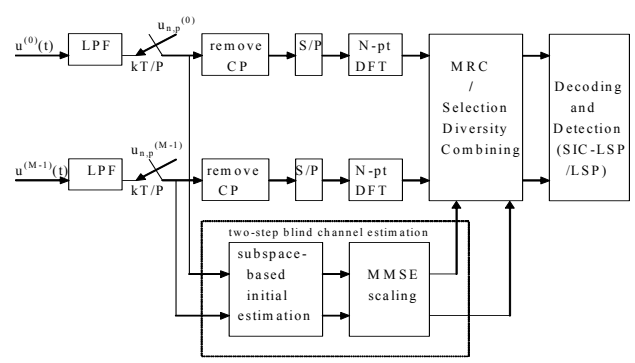

Receiver

Fig.1. A multi-user OFDM transmission system with receiver diversity.

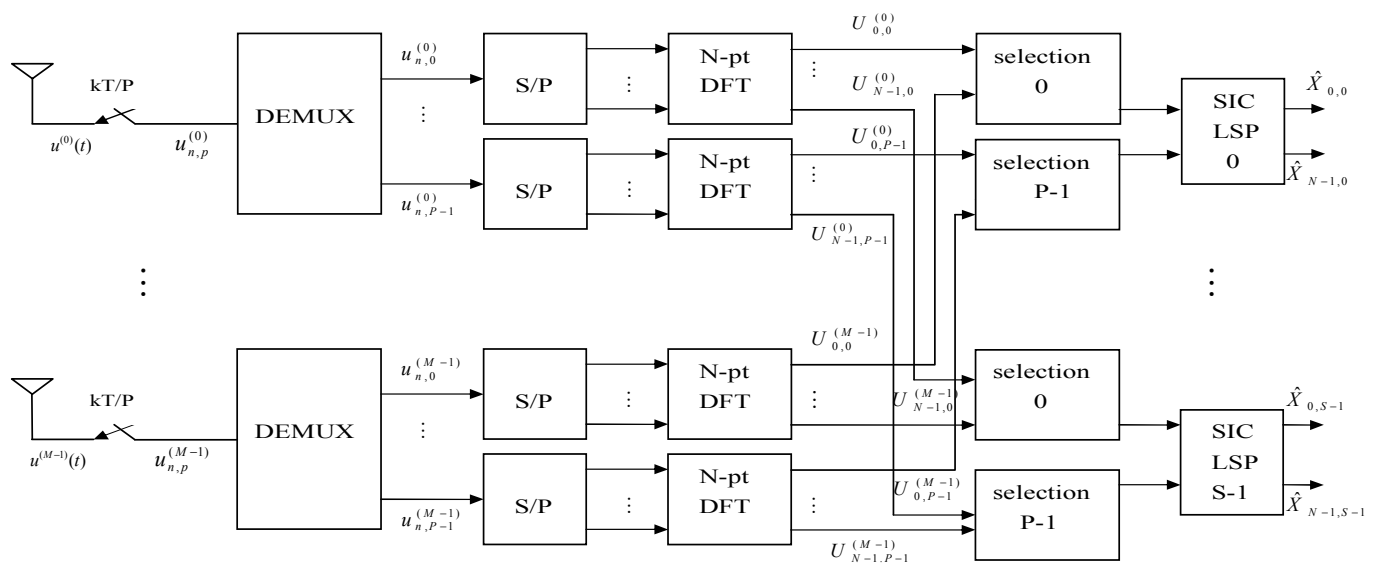

Fig.2 The MAP SDC. 


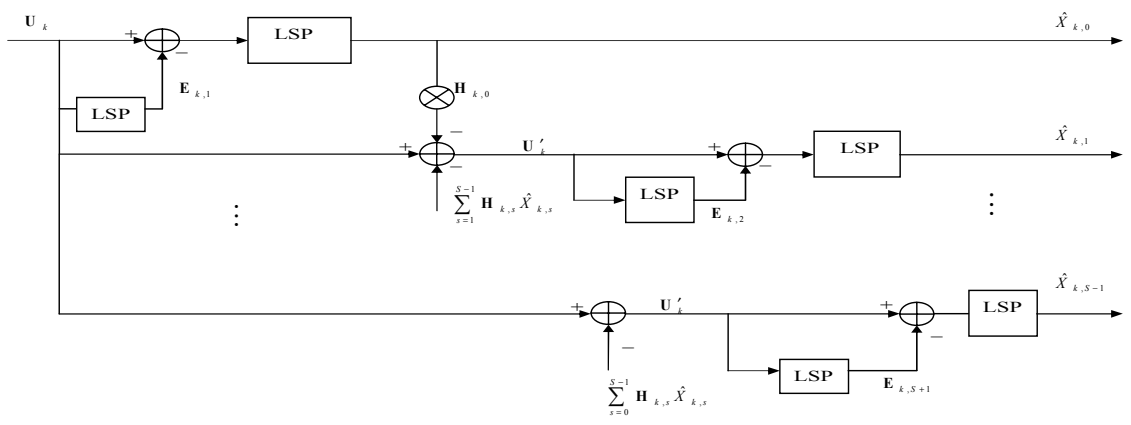

Fig.3 The entire procedure for SIC-LSP.

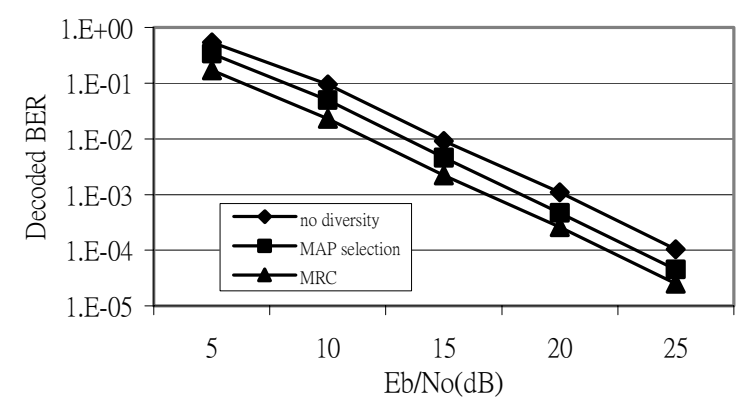

Fig.4 Comparison of the system performance for different diversity techniques in TU channel (single user).

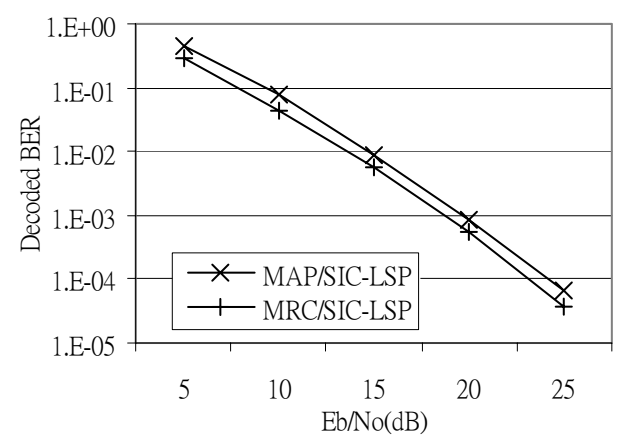

(a)

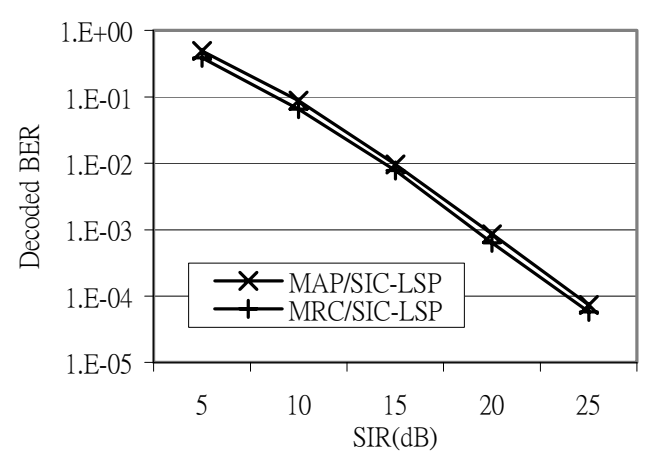

(b)

Fig.5 Comparison of the system performance for different diversity techniques in TU channel:

(a) BER versus $E_{b} / N_{0}$ and (b) BER versus SIR.

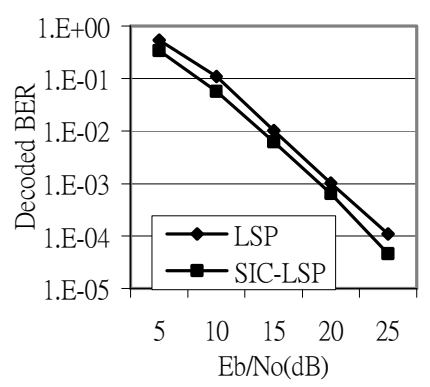

(a)

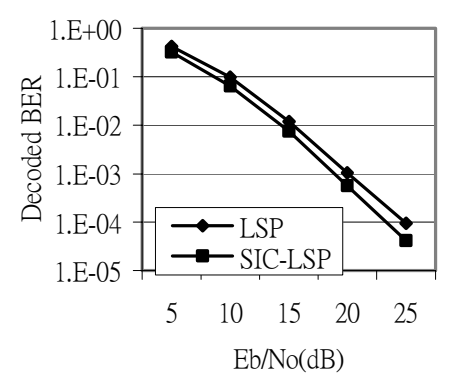

(b)

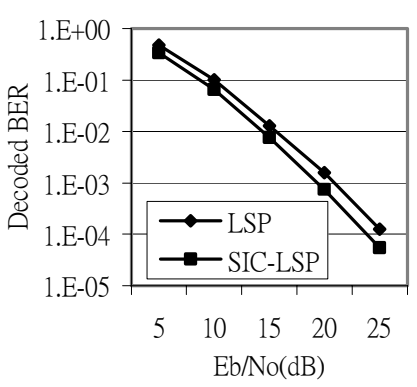

(c)

Fig.6. Comparison of the system performance (BER versus $E_{b} / N_{0}$ ) for different signal detection algorithms

(a)user1(b)user2 (c)user3. 